\title{
Exigências térmicas e estimativas do número de gerações de ortézia dos citros criadas em limão-cravo
}

\author{
Ademir Diniz Neves ${ }^{(1)}$, Marinéia de Lara Haddad ${ }^{(1)}$, Neide Graciano Zério (1) \\ e José Roberto Postali Parra (1)
}

(1)Universidade de São Paulo, Escola Superior de Agricultura Luiz de Queiroz, Departamento de Entomologia e Acarologia, Caixa Postal 09, CEP 13418-900 Piracicaba, SP. E-mail: ademir.neves@gmail.com, mlhaddad@esalq.usp.br, ngzerio@esalq.usp.br, jrpparra@esalq.usp.br

\begin{abstract}
Resumo - O objetivo deste trabalho foi determinar as exigências térmicas para o desenvolvimento de ortézia dos citros (Praelongorthezia praelonga) e estimar o número de gerações por ano nos municipios de Bauru, Barretos, São José do Rio Preto, Bebedouro, Avaré, Araraquara, Itapetininga e Limeira, no Estado de São Paulo. Para a determinação da duração das fases de ovo e ninfa, e de suas exigências térmicas, criaram-se insetos em mudas de limão-cravo (Citrus limonia) a 18, 20, 22, 25, 28, 30 e $32^{\circ} \mathrm{C}$, umidade relativa de $70 \pm 10 \%$ e fotofase de 14 horas. A duração das fases de desenvolvimento e do ciclo biológico (ovo-adulto) foi afetada pela temperatura, tendo sido maior nas temperaturas mais baixas. O limiar térmico inferior de desenvolvimento $(\mathrm{Tb})$ e a constante térmica $(\mathrm{K})$ para as fases ovo, ninfa e período ovo-adulto foram $6,16^{\circ} \mathrm{C}$ e 398,40 graus-dia (GD), $12,10^{\circ} \mathrm{C}$ e $374,50 \mathrm{GD}$, e $10,28^{\circ} \mathrm{C}$ e $735,29 \mathrm{GD}$, respectivamente. Com base nas médias de temperatura do ar dos diferentes municípios avaliados, o número de gerações por ano varia de 4,99 a 6,60.
\end{abstract}

Termos para indexação: Praelongorthezia praelonga, citros, desenvolvimento, temperatura.

\section{Temperature requirements and generation number estimates of croton mealybug reared in Rangpur lime}

\begin{abstract}
The objective of this work was to determine the temperature requirements for the development of croton mealybug (Praelongorthezia praelonga) and to estimate the number of year generations at the state of São Paulo, Brazil, counties of Bauru, Barretos, São José do Rio Preto, Bebedouro, Avaré, Araraquara, Itapetininga and Limeira. In order to determine stage durations of egg and nymph stages, and their temperature requirements, the insects were reared on Rangpur lime (Citrus limonia) seedlings at 18, 20, 22, 25, 28, 30 e $32^{\circ} \mathrm{C}$, under $70 \pm 10 \%$ relative humidity and a 14 -hour photofase. Developmental stages and biological cycle (egg-adult) durations were affected by temperature, and higher values of these parameters were obtained at lower temperatures. The lowest temperature development threshold (TT) and thermal constant (K) for egg and nymph stages and for the egg-adult period were $6.16^{\circ} \mathrm{C}$ and 398.40 degree-days (DD), $12.10^{\circ} \mathrm{C}$ and 374.50 DD, and $10.28^{\circ} \mathrm{C}$ and $735.29 \mathrm{DD}$, respectively. Based on the air temperature average values from the evaluated counties, the number of generations per year varies from 4.99 to 6.60 .
\end{abstract}

Index terms: Praelongorthezia praelonga, citrus, development and temperature.

\section{Introdução}

A cochonilha ortézia dos citros - Praelongorthezia praelonga Douglas, 1891 (Hemiptera: Ortheziidae) -, também chamada de piolho-branco, é de origem neotropical, onde é referida como infestante de plantas silvestres e ornamentais (Miller et al., 2005). Essa cochonilha tem sua sobrevivência favorecida por apresentar grande número de hospedeiros alternativos, como Coleus sp., Acalypha sp., Hibiscus sp., Patchystachys lutea (camarão-amarelo), Croton sp. e Bougainvillea sp., além de outras espécies de interesse agrícola e plantas daninhas (Suplicy Filho et al., 1983). Destaca-se, também, como praga em paisagismo e arborização urbana, sobretudo em locais sombreados (Garcia, 1999).

O primeiro registro de ocorrência dessa cochonilha no Brasil foi feito em 1900 nos estados do Pará e São Paulo, sobre Citrus limetta (Cockerel) e Hyptis sp. (Hempel), respectivamente. Em 1938, a ocorrência desse inseto foi registrada em Pernambuco (Kogan, 1964). Costa Lima (1942) alertava que essa cochonilha poderia se tornar uma praga de importância agrícola; tendo sido constatada logo após, em 1947, no Rio de Janeiro (Gonçalves \& Cassino, 1978), onde é considerada praga de importância econômica que 
causa perdas de 50 a $90 \%$ em citros. Cassino et al. (1991) relataram que essa praga é comum nesse Estado, e muitas vezes causa perdas totais em plantas cítricas. Em São Paulo, a espécie foi registrada nos pomares cítricos do Município de Severínia, em 1978 (Gonçalves \& Cassino, 1978). Atualmente, ela está disseminada em todos os estados brasileiros produtores de citros (Teixeira et al., 2001; Benvenga et al., 2004).

Ao se estabelecer na planta hospedeira, as cochonilhas sugam continuamente a seiva (Neves et al., 2006). Ocorrem também danos pela introdução do aparato bucal da cochonilha na planta, o que causa rompimento de vasos e células (Miller et al., 2005). A inserção do aparato bucal e a sucção contínua de seiva promovem a injeção de toxinas provenientes da saliva do inseto, num processo de fitotoxemia (Leite \& Pascholati, 1995) que acarreta a desfolha da planta, o enfraquecimento das árvores e a queda dos frutos. Os que não caem, comumente ficam "aguados", com baixo teor de sólidos solúveis e acidez titulável. Em casos extremos, os frutos ficam pequenos e inadequados para o comércio, seja para o consumo in natura, seja para a indústria (Teixeira et al., 2001). A cochonilha provoca ainda dano indireto, porém intenso, que é a presença do fungo Capnodium sp., conhecido como fumagina, que se desenvolve na secreção açucarada exudada ("honeydew") e afeta a fotossíntese e a transpiração das plantas (Guirado et al., 2001). O controle químico é realizado pela utilização de organofosforados granulados sistêmicos, piretroides ou inseticidas neonicotinoides (Fernandes et al., 2007).

Em razão da dificuldade de criação e lento desenvolvimento desse inseto, há poucos trabalhos sobre sua bioecologia, e as informações contidas na literatura são controversas e limitadas.

O objetivo deste trabalho foi determinar as exigências térmicas para o desenvolvimento de Praelongorthezia praelonga e estimar o número de gerações por ano nos municípios de Bauru, Barretos, São José do Rio Preto, Bebedouro, Avaré, Araraquara, Itapetininga e Limeira, no Estado de São Paulo.

\section{Material e Métodos}

O trabalho foi realizado no Laboratório de Biologia de Insetos, do Departamento de Entomologia e Acarologia, da Escola Superior de Agricultura Luiz de Queiroz, Universidade de São Paulo (Esalq/USP).
Para os estudos de laboratório, uma população de Praelongorthezia praelonga foi mantida sobre mudas de limão-cravo (Citrus limonia L.), em tubetes pretos de plástico, de $20 \mathrm{~cm}$ de comprimento por $1,5 \mathrm{~cm}$ de diâmetro. Os tubetes com as cochonilhas foram colocados em câmaras climatizadas, a 18, 20, 22, 25, 28, 30 e $32^{\circ} \mathrm{C}$, em umidade relativa de $70 \pm 10 \%$ e fotofase de 14 horas. Em cada uma das sete temperaturas, foram observadas as seguintes variáveis biológicas: duração da fase de ovo, da fase ninfal e do período ovo-adulto, que permitiram determinar as exigências térmicas para cada uma dessas fases (Haddad et al., 1999), e o número provável de gerações anuais, estimado a partir do número de graus-dia (GD) necessários para o desenvolvimento do inseto, determinado em laboratório. As temperaturas médias do ar, para o período 2001-2008, das cidades de Bauru, Barretos, São José do Rio Preto, Bebedouro, Avaré, Araraquara, Itapetininga e Limeira, no Estado de São Paulo, foram obtidas junto ao Setor de Física do Ambiente Agrícola, do Departamento de Engenharia de Biossistemas da Esalq/USP.

Para a avaliação da duração da fase de ovo, em razão de a postura ser críptica, retirou-se, a cada temperatura, uma alíquota de 50 fêmeas por dia que tiveram os ovissacos abertos para verificação da presença de ovos em seu interior; o dia foi considerado o dia inicial da oviposição. De maneira análoga, a viabilidade dos ovos foi obtida mediante abertura do ovissaco de fêmeas mortas após a oviposição, e foi feita a contagem do número de ovos remanescentes em seu interior. Utilizou-se o delineamento experimental inteiramente casualizado, com sete tratamentos de temperaturas de câmaras climatizadas e 50 repetições, cada uma delas constituída de um inseto, colocado em número variável de tubetes por tratamento.

Os dados obtidos foram submetidos à análise da variância, e as médias foram comparadas pelo teste de Tukey a $5 \%$ de probabilidade, pelo SAS. A velocidade de desenvolvimento, em razão da temperatura, foi ajustada ao modelo linear obtido pela recíproca da equação da hipérbole (Haddad et al., 1999).

\section{Resultados e Discussão}

Operíodo de incubação(Tabela 1) teve relação inversa com a temperatura, e foram constatadas durações de 13,59 dias a $32^{\circ} \mathrm{C}$ e de 32,23 dias a $18^{\circ} \mathrm{C}$. Lago (1981) constatou período de incubação de $P$. praelonga de 
37,63 dias, em Solanum tuberosum, e de 29,55 dias em Alternanthera dentata f. rubiginosa (sempre-viva), a $27 \pm 4^{\circ} \mathrm{C}$. Esses valores são, respectivamente, maior do que o encontrado a $18^{\circ} \mathrm{C}$, e semelhante ao observado a $20^{\circ} \mathrm{C}$, para $P$. praelonga em mudas de limão-cravo. Tais diferenças devem-se, em parte, ao hospedeiro utilizado e à metodologia empregada em cada situação.

$\mathrm{O}$ efeito da temperatura e do hospedeiro sobre o desenvolvimento da praga foi relatado por alguns autores. Nakano et al. (1974) observaram em Orthezia insignis Browne, mantida em brotações de S. tuberosum, um período de incubação de 24 dias à temperatura de $22^{\circ} \mathrm{C}$, semelhante ao encontrado no presente trabalho. Narai \& Murai (2002) descreveram para a cochonilha Planococcus kraunhiae (Kuwana), mantida em plantas de feijão entre 24 e $25^{\circ} \mathrm{C}$, os períodos de incubação de 10 e 8 dias, respectivamente. Em folhas de citros, Arai (1996) observou período de incubação, a $25^{\circ} \mathrm{C}$, de 29,6 dias para essa cochonilha.

Chong et al. (2008) também relataram efeito inverso da temperatura sobre o período embrionário de Maconellicoccus hirsutus (Green), que variou de 6,3 dias a $35^{\circ} \mathrm{C}$ até 16 dias a $20^{\circ} \mathrm{C}$. Neste caso, pôde-se constatar que o período de incubação desta cochonilha é relativamente curto, em comparação aos períodos observados para $P$. praelonga em limão-cravo.

Também foram descritos períodos curtos de incubação, de 8,5 e de 4 dias a $25^{\circ} \mathrm{C}$, para as cochonilhas Paracoccus marginatus Willian \& Granara de Willink e Planococcus citri (Risso), em diversos hospedeiros (Amarasekare et al., 2008; Morandi Filho et al., 2008). Entre as cochonilhas citadas anteriormente, observou-se que, em mudas de limão-cravo, P. praelonga apresenta, aparentemente, os maiores períodos de incubação.
Com relação à duração do período ninfal (Tabela 1), pôde-se observar que ocorreu relação inversa entre a duração e o aumento de temperatura, de 53,42 dias a $18^{\circ} \mathrm{C}$, e de 16,44 dias a $32^{\circ} \mathrm{C}$. Lago (1981) observou, em $P$. praelonga, período de desenvolvimento de 30,97 dias a $27 \pm 4^{\circ} \mathrm{C}$, mantida sobre brotações de S. tuberosum, enquanto Lima (1981) observou a duração da fase ninfal de $P$. praelonga de 35,16 dias a $23 \pm 3,7^{\circ} \mathrm{C}$, em batatas 'Ômega'. Em ambos os casos, os períodos apresentados são relativamente longos, o que é evidência do desenvolvimento lento dessa cochonilha.

Nakano et al. (1974) observaram para O. insignis a duração de 84,3 dias de período ninfal, quando mantida em brotações de batata 'Ômega', entre 7 e $35^{\circ} \mathrm{C}$, período também considerado longo em comparação aos de outras cochonilhas.

De maneira análoga, o ciclo total de desenvolvimento (Tabela 1) também foi afetado pela temperatura, pois houve relação inversa entre o aumento de temperatura e a duração do desenvolvimento, de 85,65 dias a $18^{\circ} \mathrm{C}$, e 30,03 dias a $32^{\circ} \mathrm{C}$. Nakano et al. (1974) descreveram um ciclo de 108,3 dias para $O$. insignis.

Lago (1981) observou período de desenvolvimento de 68,66 e 60,9 dias para $P$. praelonga, em brotações de batata e $A$. dentata f. rubiginosa, respectivamente, a $27^{\circ} \mathrm{C}$. Comparativamente, no presente trabalho, na temperatura de $28^{\circ} \mathrm{C}$ foi observado o período de desenvolvimento de 49,60 dias. Esses valores mostram a capacidade de adaptação deste inseto a diferentes temperaturas e hospedeiros. De acordo com Laflin \& Parrella (2004), as diferenças apontadas entre os diversos parâmetros biológicos são atribuídas à metodologia utilizada pelos diversos autores, ao tipo de espécie estudada, ao hospedeiro, a fatores abióticos,

Tabela 1. Duração média e intervalo de variação, em dias, das fases de ovo, ninfal e do período de ovo-adulto de Praelongorthezia praelonga em mudas de limão-cravo, submetida a diferentes temperaturas, com umidade relativa do ar de $70 \pm 10 \%$ e fotofase de 14 horas $^{(1)}$.

\begin{tabular}{|c|c|c|c|c|c|c|}
\hline \multirow[t]{2}{*}{ Temperatura $\left({ }^{\circ} \mathrm{C}\right)$} & \multicolumn{2}{|c|}{ Fase de ovo } & \multicolumn{2}{|c|}{ Fase ninfal } & \multicolumn{2}{|c|}{ Ciclo biológico } \\
\hline & Duração & Intervalo & Duração & Intervalo & Duração & Intervalo \\
\hline 18 & $32,23 \pm 0,16 a$ & $29-35$ & $53,42 \pm 0,21 \mathrm{a}$ & $49-57$ & $85,65 \pm 0,32 \mathrm{a}$ & $78-90$ \\
\hline 20 & $28,08 \pm 0,11 b$ & $26-29$ & $45,50 \pm 0,21 b$ & $42-49$ & $73,58 \pm 0,28 \mathrm{~b}$ & $68-78$ \\
\hline 22 & $23,99 \pm 0,10 \mathrm{c}$ & $21-25$ & $37,32 \pm 0,14 \mathrm{c}$ & $35-39$ & $61,31 \pm 0,20 c$ & $50-63$ \\
\hline 25 & $21,54 \pm 0,10 \mathrm{~d}$ & $18-22$ & $33,38 \pm 0,24 d$ & $30-36$ & $54,92 \pm 0,37 d$ & $48-58$ \\
\hline 28 & $22,18 \pm 0,14 \mathrm{~d}$ & $19-24$ & $27,42 \pm 0,23 \mathrm{e}$ & $24-30$ & $49,60 \pm 0,35 \mathrm{e}$ & $44-54$ \\
\hline 30 & $17,46 \pm 0,18 \mathrm{e}$ & $16-18$ & $21,28 \pm 0,24 \mathrm{f}$ & $19-25$ & $39,34 \pm 0,71 \mathrm{f}$ & $36-43$ \\
\hline 32 & $13,59 \pm 0,16 f$ & $11-15$ & $16,44 \pm 0,21 \mathrm{~g}$ & $14-19$ & $30,03 \pm 0,34 \mathrm{~g}$ & $25-33$ \\
\hline
\end{tabular}

${ }^{(1)}$ Média \pm erro-padrão, seguidas de letras iguais, nas colunas, não diferem entre si pelo teste de Tukey, a 5\% de probabilidade. 
como temperatura e umidade relativa do ar, e a variações existentes inter e intrapopulações de insetos.

A velocidade de desenvolvimento em função da temperatura ajustou-se ao modelo linear, obtido pela recíproca da equação da hipérbole (Haddad et al., 1999). Os valores obtidos de coeficientes de determinação $\left(\mathrm{R}^{2}\right)$ foram 0,91 , para a fase ninfal, e 0,90 , para a fase de ovo e período de ovo a adulto, o que atende, portanto, ao valor preconizado pelo método da hipérbole, que é de $\mathrm{R}^{2} \geq 0,90$ (Tabela 2).

Em relação às exigências térmicas da fase ovo, o limite inferior de desenvolvimento foi de $6,16^{\circ} \mathrm{C}$, e a constante térmica foi 398,40 GD, enquanto que, para o período de ovo a adulto, o limite térmico inferior de desenvolvimento foi de $10,28^{\circ} \mathrm{C}$ e a constante térmica de 735,29 GD. O valor relativamente baixo de temperatura base, para a fase ovo, pode estar relacionado ao fato de esta ser críptica e protegida de fatores ambientais externos, como variações bruscas de temperatura, no interior do ovissaco (Vogelgesang \& Szklarzewicz, 2001).

Quanto às ninfas, o limite térmico inferior de desenvolvimento foi de $12,1^{\circ} \mathrm{C}$, e a constante térmica foi de 374,50 GD (Tabela 2). De acordo com Honék (1996), o limite térmico inferior de desenvolvimento diminui com o aumento da latitude. Assim, espécies tropicais têm temperatura base $(\mathrm{Tb})$ maior, ao redor de $13,7^{\circ} \mathrm{C}$, em comparação àquelas que vivem em regiões subtropicais ( $\mathrm{Tb}$ de $10,5^{\circ} \mathrm{C}$ ); enquanto espécies de clima temperado têm $\mathrm{Tb}$ de $7,9^{\circ} \mathrm{C}$. No entanto, em relação a $P$. praelonga, inseto de origem neotropical, o valor de Tb para a fase de ovo a adulto obtido neste trabalho foi de $10,28^{\circ} \mathrm{C}$, próximo ao relatado para insetos de origem subtropical. Neste caso, deve-se levar em consideração que, em campo, o inseto se abriga na porção abaxial das folhas e no interior das copas, para obter microclima mais favorável, de temperatura mais amena em relação ao ambiente.

Tabela 2. Limiar térmico de desenvolvimento ( $\mathrm{Tb}$ ), constante térmica $(\mathrm{K})$ e coeficiente de determinação das fases de ovo, ninfa e período ovo-adulto de Praelongorthezia praelonga, em condições de laboratório ${ }^{(1)}$.

\begin{tabular}{lccc}
\hline Fase & $\mathrm{Tb}\left({ }^{\circ} \mathrm{C}\right)$ & $\mathrm{K}(\mathrm{GD})$ & $\mathrm{R}^{2}$ \\
\hline Ovo & 6,16 & 398,40 & 0,90 \\
Ninfa & 12,10 & 374,50 & 0,91 \\
Ovo-adulto & 10,28 & 735,29 & 0,90 \\
\hline
\end{tabular}

${ }^{(1)}$ Significativo a $5 \%$ de probabilidade. GD, graus-dia.
Neste aspecto, não só a latitude, mas também a altitude deve ser levada em consideração, para a classificação dos insetos quanto às exigências térmicas, uma vez que ocorre a diminuição da temperatura com o aumento da altitude (Nava et al., 2005). Além disso, sabe-se que alterações fisiológicas decorrentes da alimentação (Zeiss et al., 1996), e a origem geográfica também influenciam nessa variável (Bleicher \& Parra, 1990).

Kim et al. (2008) estudaram, em laboratório, o desenvolvimento de Pseudococcus cryptus Hempel em diferentes temperaturas e encontraram $\mathrm{Tb}$ de $6,9^{\circ} \mathrm{C}$, para a fase ovo desta espécie. Os autores relataram, para a fase imatura, a $\mathrm{Tb}$ de $8^{\circ} \mathrm{C}$, para o período ovo adulto, a $\mathrm{Tb}$ de $7,4^{\circ} \mathrm{C}$, em que as constantes térmicas foram 321,6, 506,3 e 822,9 GD para as fases ovo, ninfa e ovo-adulto, respectivamente. Em P. cryptus mantidas sobre o mesmo hospedeiro (citros 'Mandarin'), Arai (1996) observou, para a fase imatura, a $\mathrm{Tb}$ de $10^{\circ} \mathrm{C}$. A diferença de $2,0^{\circ} \mathrm{C}$ entre as $\mathrm{Tb}$ da fase imatura observada pelos diferentes autores resultou, provavelmente, do número de tratamentos (temperaturas), observações (repetições) realizadas, do tipo de gaiola utilizada (placa de Petri de diferentes diâmetros) e do tamanho e idade do disco foliar, oferecido como alimento aos insetos.

Com base nas exigências térmicas e nas médias de temperatura do ar das oito regiões citrícolas do Estado de São Paulo (Tabela 3), o número provável de gerações do inseto, ao longo do ano, varia de 4,99 a 6,60. As cidades de Bauru, Barretos, São José do Rio Preto e Bebedouro propiciaram o maior número de gerações anuais de $P$. praelonga, por serem as mais quentes, com valores de 6,60, 6,03, 5,94 e 5,35, respectivamente. As cidades de clima mais ameno, como Avaré, Araraquara, Itapetininga e Limeira

Tabela 3. Acúmulo anual de graus-dia e número provável de gerações de Praelongorthezia praelonga, em oito regiões citrícolas do Estado de São Paulo, com base na constante térmica.

\begin{tabular}{lcc}
\hline Cidade & \multicolumn{2}{c}{ Período ovo-adulto } \\
\cline { 2 - 3 } & Graus-dia anual & Número de gerações por ano \\
\hline Bauru & 4857,5 & 6,60 \\
Barretos & 4436,4 & 6,03 \\
São José do Rio Preto & 4374,3 & 5,94 \\
Bebedouro & 3941,0 & 5,35 \\
Avaré & 3853,1 & 5,24 \\
Araraquara & 3782,4 & 5,14 \\
Itapetininga & 3764,4 & 5,11 \\
Limeira & 3670,2 & 4,99 \\
\hline
\end{tabular}


apresentaram, respectivamente, 5,24, 5,14, 5,11 e 4,99 gerações anuais do inseto. Na prática, entre as cidades mais quentes e mais frias, observou-se apenas a diferença de uma geração por ano. Marin-Loayza (1999) relatou que Selenaspidus articulatus (Morgan) teve de seis a dez gerações anuais nas cidades de Limeira, São José do Rio Preto, Bebedouro, Barretos e Araraquara, enquanto Pinto (1995) avaliou, para essa mesma cochonilha, a ocorrência de quatro gerações por ano na região de Taquaritinga (SP).

Borchert et al. (2005), com base nos dados de Tb e nas normais térmicas dos EUA, concluíram que $M$. hirsutus, em razão do período extremo de inverno, conseguiria completar apenas uma geração por ano, em todo território.

\section{Conclusões}

1. A menor temperatura de base, $6,16^{\circ} \mathrm{C}$, para o desenvolvimento de Praelongorthezia praelonga ocorre na fase de ovo, e a constante térmica para o ciclo total é de 735,29 GD.

2. A determinação das exigências térmicas para o desenvolvimento de Praelongorthezia praelonga permitem estimar a ocorrência de 4,99 a 6,60 gerações da praga por ano, nos principais municipios citrícolas do Estado de São Paulo.

\section{Referências}

AMARASEKARE, K.G.; CHONG, J.H.; EPSKY, N.D.; MANNION, C.M. Effect of temperature on the life history of the mealybug Paracoccus marginatus (Hemiptera: Pseudococcidae). Journal of Economic Entomology, v.101, p.1798-1804, 2008.

ARAI, T. Temperature-dependent developmental rate of three mealybug species, Pseudococcus citriculus Green, Planococcus citri (Risso), and Planococcus kraunhiae (Kuwana) (Homoptera: Pseudococcidae) on citrus. Japanese Journal of Applied Entomology and Zoology, v.40, p.25-34, 1996.

BENVENGA, S.R.; GRAVENA, S.; SILVA, J.L. da; ARAUJO JUNIOR, N.; AMORIM, L.C.S. Manejo prático da cochonilha ortézia em pomares de citros. Laranja, v.25, p.291-312, 2004.

BLEICHER, E.; PARRA, J.R.P. Espécies de Trichogramma parasitóides de Alabama argillacea. III: Determinação das exigências térmicas de três populações. Pesquisa Agropecuária Brasileira, v.25, p.215-219, 1990.

BORCHERT, D.; NIETSCHKE, B.; FIESELMANN, D. Pink hibiscus mealybug ad hoc report. Washington: Raleigh: USDA, 2005.9p.

CASSINO, P.C.R.; LIMA, A.F. de; RACCA FILHO, F. Orthezia praelonga Douglas, 1891 em plantas cítricas no Brasil (Homoptera,
Ortheziidae). Arquivos da Universidade Federal Rural do Rio de Janeiro, v.14, p.35-57, 1991.

CHONG, J.H.; RODA, A.L.; MANNION, C.M. Life history of the mealybug, Maconellicoccus hirsutus (Hemiptera: Pseudococcidae), at constant temperatures. Environmental Entomology, v.37, p.323-332, 2008.

COSTA LIMA, A.M. da. Homópteros. In: COSTA LIMA, A.M. da. Insetos do Brasil. Rio de Janeiro: Escola Nacional de Agronomia, 1942. v.3, 327p.

FERNANDES, O.A.; CARNEIRO, T.R.; CAMPOS, A.P. de; OLIVEIRA, T.T. de; FERREIRA, M. da C. Dispersão de Orthezia praelonga Douglas, 1891 (Hemiptera: Ortheziidae) causada por equipamentos de pulverização em pomar de citros. Revista Brasileira de Fruticultura, v.29, p.249-253, 2007.

GARCIA, A.H. Levantamento, identificação e avaliação dos danos de insetos em árvores ornamentais na área urbana de Goiânia (GO). Pesquisa Agropecuária Tropical, v.29, p.77-81, 1999.

GONÇALVES, C.R.; CASSINO, P.C.R. O problema da Orthezia praelonga na citricultura. In: ENCONTRO NACIONAL DE CITRICULTURA, 5., 1978, Rio de Janeiro. Anais. Rio de Janeiro: Pesagro, 1978. p.5-10.

GUIRADO, N.; SAKAI, E.; AMBROSANO, E.J. Avaliação de efeito do óleo de nim extraído de sementes de Azadirachta indica no controle da cochonilha ortézia em laranja 'Pêra'. Revista de Agricultura, v.76, p.401-409, 2001.

HADDAD, M.L.; PARRA, J.R.P.; MORAES, R.C.B. Métodos para estimar os limites térmicos inferior e superior de desenvolvimento de insetos. Piracicaba: FEALQ, 1999. 29p.

HONÉK, A. Geographical variation in thermal requirements for insect development. European Journal of Entomology, v.93, p.303-312, 1996.

KIM, S.C.; SONG, J.-H.; KIM, D.-S. Effect of temperature on the development and fecundity of the cryptic mealybug, Pseudococcus cryptus, in the laboratory. Journal of Asia-Pacific Entomology, v.11, p.149-153, 2008.

KOGAN, M. Nota sobre as espécies do gênero Orthezia Bosq d'Antic, 1784, de importância econômica e que ocorrem no Brasil. Agronomia, v.22, p.134-144, 1964.

LAFLIN, H.M.; PARRELLA, M.P. Developmental biology of citrus mealybug under conditions typical of California rose production. Annals of Entomological Society of America, v.97, p.982-988, 2004.

LAGO, I.C.S. Biologia de Orthezia praelonga Douglas, 1891 (Homoptera - Ortheziidae), distribuição geográfica, plantas hospedeiras e ocorrência de inimigos naturais, em Pernambuco. 1981. 78p. Dissertação (Mestrado) - Universidade Federal Rural de Pernambuco, Recife.

LEITE, B.; PASCHOLATI, S.F. Hospedeiro: alterações fisiológicas induzidas por fitopatógenos. In: BERGAMIN FILHO, A.; KIMATI, H.; AMORIM, L. Manual de fitopatologia: princípios e conceitos. 3.ed. São Paulo: Agronômica Ceres, 1995. v.1, p.393-416.

LIMA, A.F. Bioecologia de Orthezia praelonga Douglas, 1891 (Homoptera, Ortheziidae). 1981. 126p. Dissertação (Mestrado) - Escola Superior de Agricultura Luiz de Queiroz, Universidade de São Paulo, Piracicaba. 
MARIN-LOAYZA, R.M. Bioecologia e determinação do nível de controle de Selenaspidus articulatus (Morgan, 1889) (Hemiptera: Diaspididae) em citros. 1999. 110p. Tese (Doutorado) - Escola Superior de Agricultura Luiz de Queiroz, Piracicaba.

MILLER, D.R.; MILLER, G.L.; HODGES, G.S.; DAVIDSON, J.A. Introduced scale insect (Hemiptera: Coccoidea) of the United States and their impact on U.S. agriculture. Proceedings of Entomological Society of Washington, v.107, p.123-158, 2005.

MORANDI FILHO, W.J.; GRÜTZMACHER, A.D.; BOTTON, M.; BERTIN, A. Biologia e Tabela de vida de fertilidade de Planococcus citri em diferentes estruturas vegetativas de cultivares de videira. Pesquisa Agropecuária Brasileira, v.43, p.941-947, 2008.

NAKANO, O.; JOKO, T.; PARRA, J.R.P. Observações sobre a biologia da Orthezia insignis Browne, 1887 (Homoptera-Ortheziidae). Anais da Sociedade Entomológica do Brasil, v.3, p.44-48, 1974.

NARAI, Y; MURAI, T. Individual rearing of the mealybug, Planococcus kraunhiae (Kuwana) (Homoptera: Pseudococcidae) on germinated broad bean seeds. Applied Entomology and Zoology, v.37, p.295-298, 2002.

NAVA, D.E.; HADDAD, M. de L.; PARRA, J.R.P. Exigências térmicas, estimativa do número de gerações de Stenoma catenifer e comprovação do modelo de campo. Pesquisa Agropecuária Brasileira, v.40, p.961-967, 2005.
NEVES, A.D.; OLIVEIRA, R.F.; PARRA, J.R.P. A new concept for insect damage evaluation based on plant physiological variables. Anais da Academia Brasileira de Ciências, v.78, p.821-835, 2006.

PINTO, A. de S. Flutuação populacional de Selenaspidus articulatus (Morgan) Hemiptera: Diaspididae) em citros e ocorrência de inimigos naturais no município de Taquaritinga, SP. 1995. 95p. Dissertação (Mestrado) - Universidade Estadual Júlio Mesquita Filho, Jaboticabal.

SUPLICY FILHO, N.; SAMPAIO, A.S.; MYAZAKI, I. Considerações sobre o coccídeo Orthezia praelonga Douglas, 1891, importante praga da citricultura brasileira. O Biológico, v.49, p.19-24, 1983.

TEIXEIRA, M.A.; BETTIOL, W.; CESNIK, R. Patogenicidade do fungo Colletotrichum gloeosporioides, patógeno de Orthezia praelonga, para folhas, frutos e flores cítricas. Summa Phytopathologica, v.27, p.352-357, 2001.

VOGELGESANG, M.; SZKLARZEWICZ, T. Formation and structure of egg capsules in scale insects (Hemiptera, Coccinea). I. Ortheziidae. Arthropod Structure \& Development, v.30, p.63-68, 2001.

ZEISS, M.R.; KOEHLER, K.J.; PEDIGO, L.P. Degree-day requirements for development of the bean leaf beetle (Coleoptera: Chrysomelidae) under two rearing regimes. Journal of Economic Entomology, v.89, p.111-118, 1996.

$\overline{\text { Recebido em } 4 \text { de abril de } 2010 \text { e aprovado em } 22 \text { de junho de } 2010}$ 